\title{
VARIABILITÉ GÉNÉTIQUE DE BARBUS MÉRIDIONALIS RISSO (CYPRINIDAE) : UNE ESPËCE QUASI MONOMORPHE
}

\author{
P. BerRebI (1), G. LAMY (2), Ghislaine CATTANeO-BerRebI (1), J.F. RENNo (1)
}

(1) Laboratoire de Génétique de l'Institut des Sciences de l'Evvolution (CNRS, UA 327), Université de Montpellier II, Place E. Bataillon - 34060 MONTPELLIER Cedex, France.

(2) Parc National des Cévennes - 48400 FLORAC, France.

Reçu le 22 juin 1987

Received 22 June, 1987

Accepté le 12 avril 1988

Accepted 12 April, 1988

\section{RÉSUMÉ}

Barbus meridionalis a été échantillonné dans 5 petits cours d'eau de moyenne altitude dans les Pyrénées, le Massif Central et les Alpes. Le preferendum écologique de l'espèce est précisé. L'étude électrophorétique des allozymes de 34 locus a montré une absence quași totale de polymorphisme. Les seules exceptions ont été observées dans le Tech (Pyrénées Orientales) où 3 locus sont polymorphes, et dans un ruisseau du Massif central où un allèle nul est présent. L'hypothèse d'un effet fondateur lié aux dernières glaciations est proposée pour expliquer ces observations.

Mots clefs : Barbus, Cyprinidae, Polymorphisme enzymatique, Effet fondateur, Glaciation, Spéciation, Biogéographie.

\section{SUMMARY}

\section{GENETIC VARIABILITY OF BARBUS MERIDIONALIS RISSO (CYPRINIDAE) : A NEARLY MONOMORPHIC SPECIES}

Barbus meridionalis was sampled in five small rivers of middle altitude in three French mountains : the Pyrenees, the Massif Central and the Alps. The ecological preferendum of the species is discussed. The electrophoretic study of the allozyms of 34 loci shows an almost total lack of polymorphism. Few exceptions were observed in the Tech (Eastern Pyrenees) where three loci are polymorphic, and a stream of the Massif Central where a null allele is present at a high frequence. An hypothesis including a bottleneck by founder effect caused by the last glaciation is proposed.

Key Words: Barbus, Cyprinidae, Enzymatic polymorphism, Founder effect, Glaciation, Speciation, Biogeography.

\section{INTRODUCTION}

Barbus meridionalis appartient à la famille des Cyprinidés (Figure 1). II se distingue des autres espèces européennes du même genre par une forme plus trapue, de grandes écailles, une nageoire anale très longue, une coloration dorée ponctuée de noir et surtout par l'absence totale de denticulations sur le dernier rayon dur de la nageoire dorsale, elle-même de profil convexe. Enfin, il se limite aux régions méditerranéennes de l'Europe et au Nord-ouest de la mer Noire.

Sa répartition géographique est assez morcelée. La systématique de l'espèce a été fortement controversée (KOLLER, 1926 ; BERG, 1932 ; BANARESCU, 1964 ; LADIGES et al., 1965; ALMAÇA 1968 et 1971; STEPHANIDIS, 1971; KARAMAN, 1971; VUKOVIC et al., 1971). La division en sous-espèces qui nous semble la plus argumentée est celle d'ALMAÇA (1984) avec trois sousespèces:

B. m. meridionalis se rencontre au Nord de l'Espagne, au Sud de la France.

B. $m$. petenyi occupe la Tchécoslovaquie, la Pologne et la Roumanie (bassins du Danube, de la Vistule et du Dniestr).

B. m. peloponnesius habite la Grèce du Sud.

II faut ajouter à cela les populations de l'Albanie et du Nord de la Grèce à caractères intermédiaires petenyi-peloponnesius.

Une étude génétique basée sur l'électrophorèse des protéines enzymatiques apporterait des éléments nouveaux. En effet, ce type d'étude s'affranchit des cas d'écomorphes et utilise des marqueurs dont on est sûr du déterminisme génétique. 


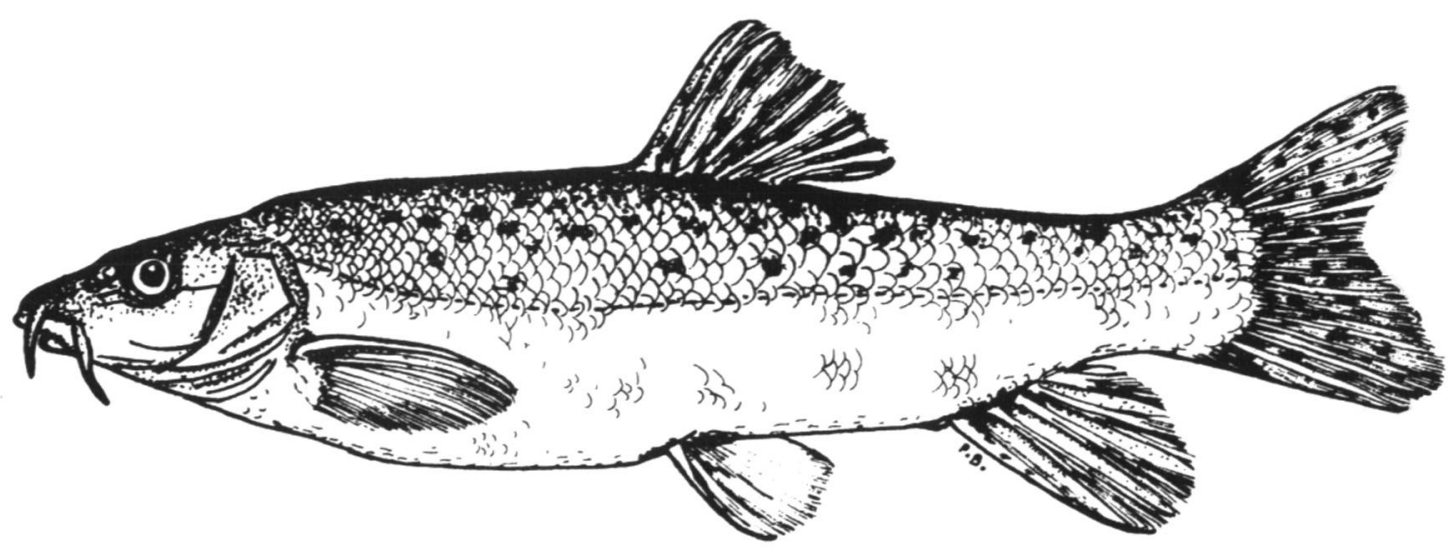

Figure 1 : Barbus meridionalis RISSO.

Figure 1 : Barbus meridionalis RISSO

La présente étude concerne les populations françaises de la sous-espèce meridionalis. Dans cette partie occidentale de son aire de répartition, le barbeau méridional vit dans de petits cours d'eau, généralement de moyenne altitude (SPILLMAN, 1961). II se concentre, en France, dans trois grandes zones: les Pyrénées Orientales, le Sud du Massif Central et le Sud-Ouest des Alpes.

Une seule étude cytologique a été entreprise sur cette espèce. Selon SOFRADZIJA et al. (1973), la sous-espèce petenyi de Yougoslavie possède 100 chromosomes. II s'agit donc bien d'une espèce tétraploïde, comparable à Barbus barbus étudié par WOLF et al. (1969) et HAFEZ (1981). Ce nombre de 100 est environ le double de celui des Cyprinidés diploïdes (48 à 50 chromosomes selon HAFEZ, 1981). Enfin, d'après l'étude de TRIANTAPHYLLIDIS et al. (1981), la nature tétraploïde de l'espèce est confirmée (du moins pour la sous-espèce peloponnesius) par les duplications de locus pour les systèmes Ldh et Pgm.

Avant de commencer l'étude de l'espèce sur l'ensemble de sa répartition, il nous semble important de connaître la variabilité de sa sous-espèce française sur une trentaine de locus enzymatiques. Ainsi, les trois formations montagneuses où la sous-espèce se rencontre en France (zone centrale de sa répartition) ont été prospectées.

\section{MATÉRIELS ET MÉTHODES}

Les localités prospectées sont précisément indiquées sur la figure 2. Quelques caractéristiques écologiques qui nous semblent fondamentales ont été relevées et exposées dans le tableau I. Ces quelques observations ont pour but de définir plus précisément le preferendum écologique de l'espèce, compte tenu de l'idée généralement admise que l'altitude est le paramètre essentiel liéà la présence du barbeau méridional. Afin d'étayer les hypothèses émises plus bas, deux cours d'eau de plaine, la Boyne de la Dourbie (Station 1 et 2 sur la figure 2,) peuplés de barbeaux méridionaux ont été inclus dans l'étude écologique sans être échantillonnés. L'échantillonnage concerne seulement de petits ruisseaux situés dans les trois massifs montagneux. Les poissons sont capturés par pêche électrique au nombre de 36 par cours d'eau. Transportés vivants au laboratoire, il sont congelés à $-30^{\circ} \mathrm{C}$. Lors de la dissection, 0,5 à 2 grammes de muscle et de foie sont prélevés et broyés en présence d'une quantité égale de tampon Tris-HCl-EDTA-NADP à pH 6,8. Après centrifugation, le surnageant est prélevé et conservé à $-60^{\circ} \mathrm{C}$.

Les électrophorèses se font sur gel d'amidon horizontal selon la technique de MORETTI et al. (1957). Les colorations sont celles de SHAW et al. (1970), les tampons sont ceux de PASTEUR et al. (1985).

Les électromorphes, assimilés à des allèles, sont numérotés par ordre de rapidité électrophorétique croissante, d'abord en comptant les locus codant pour des enzymes à migration anodique, puis cathodique. Les locus actifs seulement dans le muscle sont notés $M$; actifs uniquement dans le foie, ils sont notés F; actifs dans les 2 organes, ils n'ont pas de suffixe. Les électromorphes les plus fréquents sont nommés 100 , les autres sont numérotés en fonction de leur position relative en fin de migration. La fréquence de l'allèle nul de Aat-1, a été calculée comme la racine carrée de la fréquence des individus n'ayant aucune activité, et considérés comme homozygotes pour cet allèle nul. 


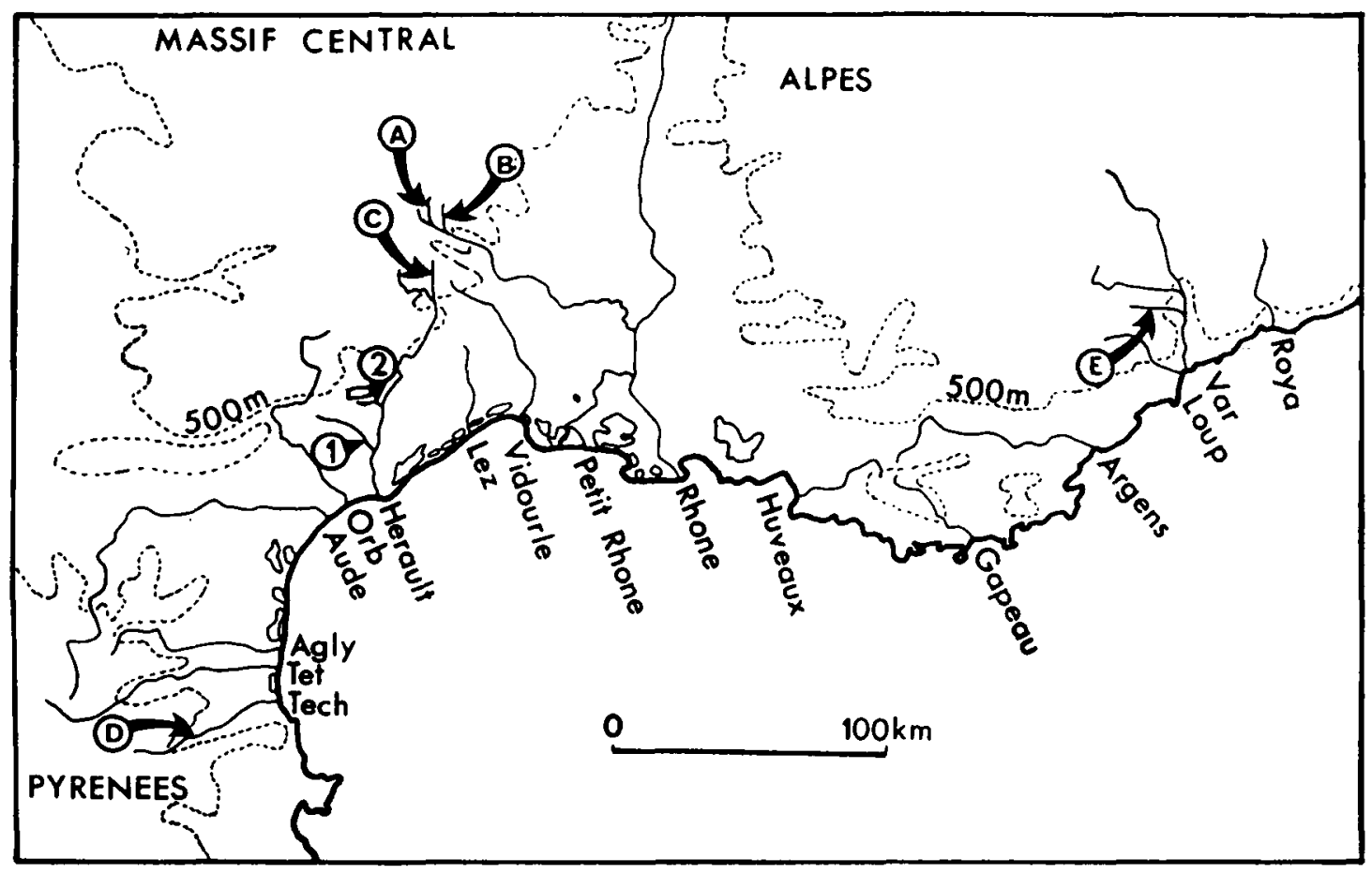

Figure 2 : Stations échantillonnées $(A=$ Terrades, $B=$ Pradet, $C=$ Rieutord, $\mathbf{D}=$ Tech, $E=$ Esteron) et stations de référence écologique ( $1=$ Boyne, $2=$ Dourbie $)$.

Figure 2 : Sampled stations ( $\mathrm{A}=$ Terrades, $\mathrm{B}=$ Pradet, $\mathrm{C}=$ Rieutord, $\mathrm{D}=$ Tech, $\mathrm{E}=\mathrm{Esteron}$ ) and ecological stations $(1=$ Boyne, $2=$ Dourbie $)$.

\section{RÉSULTATS}

Les paramètres écologiques qui nous ont semblés importants ont été résumés en tableau 1.

Tableau I : Relevé des paramètres écologiques à 7 stations dont 5 ont été échantillonnées (A àE). Table 1 : Ecological parameters in the 7 stations of which 5 were sampled (A to E).

\begin{tabular}{|c|c|c|c|c|c|c|}
\hline COUKS O EAN & STATION & AFFLUENCE & $\begin{array}{l}\text { ALTITUDE } \\
\text { STATIONV }\end{array}$ & $\begin{array}{l}\text { "COLFS D'EAN } \\
\text { ACCIDENTE" }\end{array}$ & $\begin{array}{l}\text { FRESENCE } \\
\text { DE TRUITE }\end{array}$ & $\begin{array}{l}\text { PRESENCE } \\
\text { DE B. BAFEUS }\end{array}$ \\
\hline $\begin{array}{l}\text { TERFFALES } \\
\text { PFAGDET }\end{array}$ & $\begin{array}{l}\text { A } \\
B\end{array}$ & $\begin{array}{l}\text { GAFD } \\
\text { (FHAOLE) }\end{array}$ & $\begin{array}{l}500 \mathrm{~m} \\
450 \mathrm{~m}\end{array}$ & $\begin{array}{l}\text { OUI } \\
\text { OUI }\end{array}$ & $\begin{array}{l}\text { HON } \\
\text { NON }\end{array}$ & NON \\
\hline FIEUTCFID & C & & $60 \mathrm{~cm}$ & OUI & NON & NON \\
\hline DCUREIE & 1 & HERGULT & 50 & OUI & NON & NON \\
\hline BOYNE & 2 & & $50 \mathrm{n}$ & OUI & NON & NON \\
\hline TECH & D & TECH & 150 & NON & WUI & NON \\
\hline ESTERON & $E$ & VAR & $70 \mathrm{in}$ & OUI & OUI & NON \\
\hline
\end{tabular}


Le premier résultat évident de l'étude succincte des quelques paramètres écologiques est que seule la présence de $B$. barbus semble strictement corrélée (négativement) à la présence de $B$. meridionalis.

Le tableau II expose les fréquences alléliques au niveau de 4 locus polymorphes. Seuls les locus Adh, Fum-3 et Pgm-1 ont montré plus d'un allèle, et ce, uniquement dans la population des Pyrénées ( $P=0,09$ où $P$ est la proportion de locus polymorphes). Dans toutes les autres populations, aucun locus ne s'est révélé variant $(P=0,00)$, à l'exception de Aat-1 dans le ruisseau du Pradet (station $C, P=0,03$ ) où un allèle nul est présent avec une fréquence de 0,441 . Cette fréquence théorique a été obtenue en supposant que l'absence d'activité de ce locus correspondait à un allèle nul rècessif (donc à l'état homozygote) et que les deux allèles (actif et inactif) étaient en équilibre de Hardy-Weinberg.

Tableau II : Fréquences alléliques des 4 locus polymorphes analysés chez 36 B. meridlonalis par localité. $\mathbf{N T}=$ non traité, $\mathbf{A}=$ Terrades, $\mathbf{B}=$ Pradet, $\mathbf{C}=$ Rieutord, $\mathbf{D}=$ Tech, $\mathbf{E}=$ Esteron.

Table II : Frequence of the alleles at the 4 polymorphic loci screened (36 individuals each locality). NT $=$ not studied, $\mathbf{A}=$ Terrades station, $\mathbf{B}=$ Pradet, $\mathbf{C}=$ Rieulord, $\mathbf{D}=$ Tech, $\mathbf{E}=$ Esteron.

\begin{tabular}{|cc|c|c|c|c|c|}
\hline LOCUS Allele & STAIION A & STATION B & STATION C & STRTION D & STATION E \\
\hline Aat-1 actif & 1,00 & 0,66 & 1,00 & 1,00 & 1,00 \\
& inactif & 0,00 & 0,44 & 0,00 & 0,00 & 0,00 \\
Adh-F 100 & 1,00 & 1,00 & 1,00 & 0,96 & 1,00 \\
& 55 & 0,00 & 0,00 & 0,00 & 0,04 & 0,00 \\
& & 1,00 & 1,00 & 1,00 & 0,89 & 1,00 \\
Fun-3 100 & 0,00 & 0,00 & & 0,11 & 0,00 \\
& 88 & 1,00 & 1,00 & 1,00 & 0,64 & 1,00 \\
Pga-1 117 & 0,00 & 0,00 & 0,00 & 0,36 & 0,00 \\
\hline
\end{tabular}

Les systèmes enzymatiques monomorphes les plus complexes (c'est-à-dire les systèmes multilocus) sont interprétés à la figure 3 .

\section{DISCUSSION}

Les deux thèmes abordés, à savoir le preferendum écologique de l'espèce et son polymorphisme enzymatique nous permettent d'avancer des hypothèses sur la répartition actuelle de la sous-espèce en France.

\subsection{Preferendum écologique}

Selon SPILLMAN (1961), le barbeau méridional ne serait limité que par l'altitude (300 à $800 \mathrm{~m}$ environ) bien qu'il cite un cours d'eau de faible altitude peuplé par cette espèce. ALMAÇA (1981) considère que la forme et la structure de sa nageoire dorsale sont caractéristiques de la vie en altitude.

S'il est exact que la grande majorité des populations de cette espèce se rencontre à ces altitudes, ce n'est pas une règle générale (voir tableau I). L'altitude ne serait donc pas un paramètre agissant directement mais il définirait seulement les zones où le biotope caractéristique de l'espèce serait le plus souvent rencontré. Ces biotopes sont caractérisés par des cours d'eau de faible débit, séparés du lit principal par des zones rapides et des cascades, certaines parties sont asséchées en été ; enfin on y observe des crues décapantes une ou deux fois par an. Or, cette description ("cours d'eau accidenté") s'applique exactement aux deux cours d'eau de plaine ajoutés à l'étude: la Boyne et la Dourbie. Ce type de biotope, rapidement décrit, ne suffit pas à expliquer totalement la présence de B. meridionalis : le Tech (Pyrénées Orientales) est en effet "accidenté" dans sa partie haute, mais son cours se calme nettement dans sa partie basse. Or c'est dans cette partie basse, assez large, à débit plus important, que les barbeaux ont été collectés. 


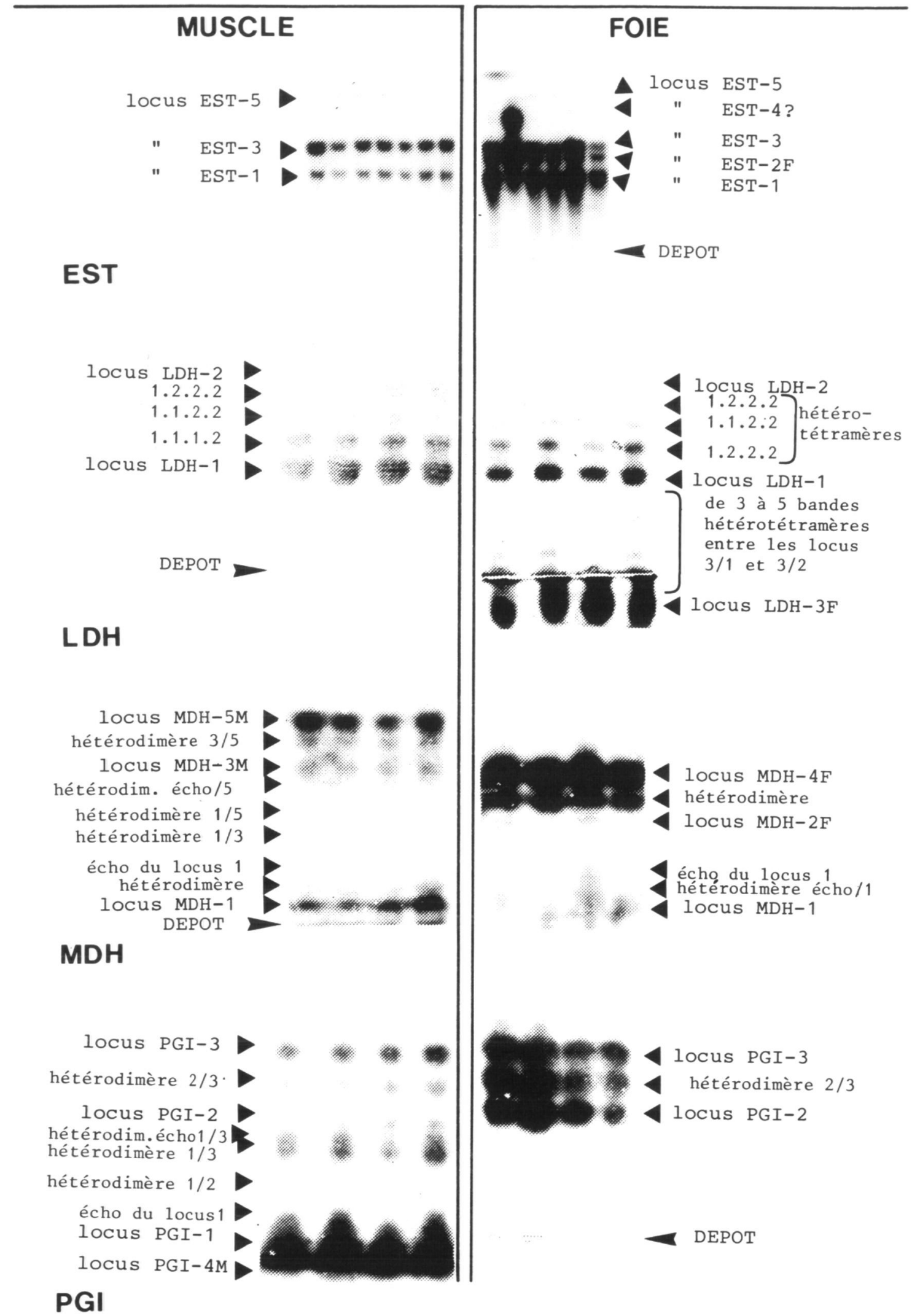

Figure 3 : Interprétation génétique des zymogrammes les plus complexes.

Figure 3 : Genetical interpretation of the most complex zymograms. 
Notons également l'absence de truites dans la partie amont des ruisseaux du Massif Central. Le régime climatique méditerranéen à fort étiage avec baisse concomittante du taux d'oxygène dissous autorise la survie du cyprinidé et pas celle du salmonidé, plus exigeant : les parties amont de ces cours d'eau se transforment en été en une suite de bassins eutrophisés. Dans les Cévennes, nous avons observé que la truite fario, souvent introduite par l'homme, paraît entraîner, par compétition (destruction des œufs et des alevins de barbeaux), la disparition de $B$. meridionalis. Cependant, la coexistence de truites et de barbeaux dans le Tech et l'Esteron n'en font pas un facteur limitant absolu.

Un autre paramètre, permet d'arriver à une interprétation cohérente: $B$. meridionalis semble éviter la présence de $B$. barbus, espèce proche de grande taille et compétiteur intragénérique. Etant seul à pouvoir coloniser les cours accidentés, le barbeau méridional peut s'y développer à l'abri de son concurrent. On le trouve fréquemment en micro-populations isolées dans la retenue la plus amont des cours d'eau des Cévennes. Ces groupes de quelques dizaines d'individus sont totalement isolés du reste de l'espèce. Dans les zones de moyenne altitude, cette concurrence est toujours évitée. En basse altitude, seuls les cours d'eau où $B$. barbus ne peut pas remonter (zones accidentées) sont habités par B. meridionalis. Quelquefois la présence simultanée des deux espèces est possible et une hybridation spontanée a lieu.

L'absence d'isolement reproductif nous conforte dans l'hypothèse selon laquelle cette compétition intragénérique est récente et que c'est par séparation écologique que les deux espèces ont divergé. ALMAÇA (1976 et 1984) considère que $B$. barbus est l'espèce la plus proche de l'ancêtre provenant de Sibérie durant l'Oligocène. B. meridionalis serait issu, par endémisme périméditerranéen, de cet ancêtre commun mais aurait colonisé le haut des rivières tout comme $B$. cyclolepis en Grèce et Bulgarie (BANARESCU, 1964). Cette spéciation parapatrique, se partageant des zones différentes d'un même cours d'eau, serait l'explication de la répartition actuelle du barbeau méridional. On peut considérer ces deux espèces comme directement issues d'un partage de niche à partir d'une espèce ancestrale.

\subsection{Polymorphisme enzymatique}

Selon Darlington (1948 et 1957), les Cyprinidés proviendraient d'Asie tropicale d'où ils ont gagné par une première voie "sibérienne" l'Europe (sous-genre Barbus) et, tardivement, l'Afrique du Nord. Par une seconde voie de migration, ils auraient atteint l'Afrique australe (sous-genre $L$ abeobarbus) et finalement l'Afrique du Nord (figure 4). Les grands caractères distinctifs de ces deux sous-genres ont été précisés par Berrebi (1981).

C'est bien sûr par la branche européenne que les ancêtres de Barbus (Barbus) meridionalis ont pénétré en Europe. D'après Dottrens (1952) et Spillman (1961), la répartition géographique de $B$. meridionalis aurait été beaucoup plus vaste quand cette espèce appartenait à la faune chaude préglaciaire.

L'étude du polymorphisme allozymique au niveau de près de 35 locus a montré une absence assez exceptionnelle de variants. Il est également remarquable que la population polymorphe (station D: le Tech, dans les Pyrénées Orientales), soit précisément située le plus au Sud et en basse altitude. D'autre part, c'est toujours l'allèle le plus fréquent des locus polymorphes de cette population que l'on retrouve fixé dans les populations d'altitude.

Selon une première hypothèse, la répartition actuelle de l'espèce serait issue de l'action des glaciations suivi d'une reconquête récente des biotopes d'altitude. Selon BANARESCU (1973), les espèces d'eau douce de la région du Danube ont été moins atteintes par les glaciations pléistocènes que celles de nos régions, ce qui explique la présence actuelle de la sous-espèce petenyi en Europe de l'Est. En Europe occidentale seules les populations de la côte méditerranéenne auraient survécu. Avec le réchauffement des 10.000 dernières années, les populations les plus au Sud et de plus basse altitude auraient enfin pu coloniser les cours d'eau de moyenne altitude. La population pyrénéenne serait donc proche des populations préglaciaires tandis que toutes les autres populations échantilIonnées du Massif Central et des Alpes seraient fondées depuis peu avec réduction extrême du polymorphisme enzymatique.

On ne peut exclure, comme seconde hypothèse, l'action de l'homme dans un passé très récent, puisque la pêche au barbeau est appréciée. Cette action peut expliquer la perte de polymorphisme par effet fondateur.

Le cas de l'allèle nul de la population du Pradet (station B) doit être interprété de toute autre manière. Tout d'abord, la fréquence calculée de 0,441 est très élevée et nettement supérieure aux fréquences observées dans le Tech (station D) pour les locus polymorphes. Ceci est à mettre en rapport avec l' "effet fondateur": si cet allèle nul, rare dans la population d'origine, se trouvait par hasard présent dans le petit nombre de fondateurs, il a acquis instantanément une fréquence respectable. Le fait qu'il ne soit pas présent dans le ruisseau voisin (station $\mathrm{C}$ ), montre bien que les échanges géniques sont très limités entre cours d'eau. Une proportion aussi élevée pour un allèle 
nul n'est pas fréquente chez les poissons. Elle est probablement en rapport avec l'état dupliqué des locus Aat-1 et Aat-2. Les deux protéines enzymatiques qu'ils codent ayant un rôle similaire, un allèle nul homozygote à l'un d'entre eux n'est pas léthal.

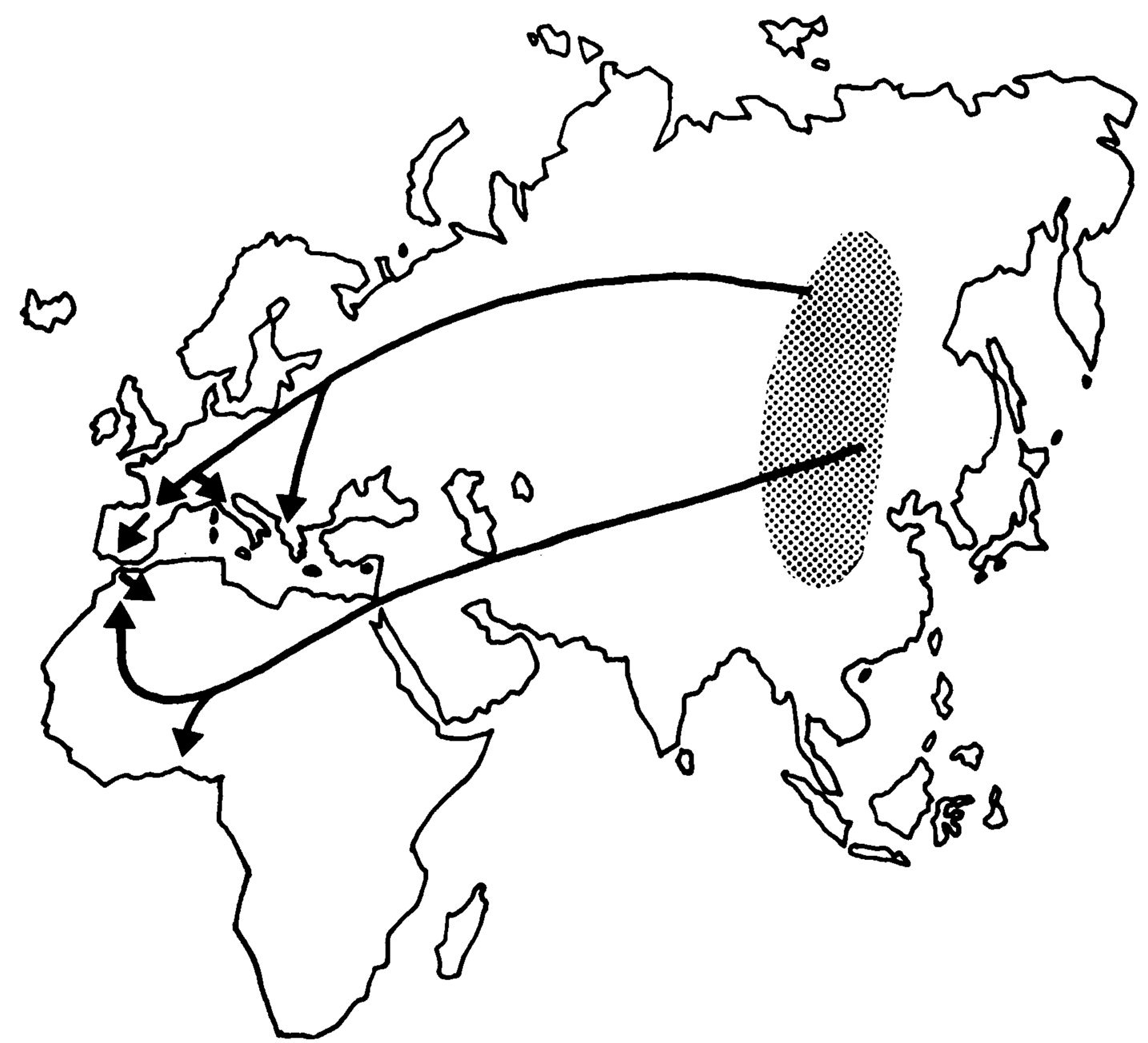

Figure 4 : Diagramme de dispersion du genre Barbus.

Figure 4 : Diagram of dispersion of the genus Barbus.

En conclusion, l'état actuel des peuplements de $B$. meridionalis ne peut être compris que par son histoire évolutive récente. Les facteurs essentiels de cette histoire semblent être les dernières glaciations et la confrontation avec l'espèce cousine, le barbeau fluviatile. Ces caractéristiques ainsi que la tétraploïdie de ces espèces en font un modèle d'un grand intérêt évolutif.

\section{REMERCIEMENTS}

Les auteurs remercient le Parc National des Cévennes, la Direction de la Protection de la Nature et le Conseil Supérieur de la Pêche pour leur aide financière. Les pêches ont été effectuées avec l'aide gracieuse de P. LIM et F. DICLARD (ENSA, Toulouse), D. FOUBERT et P. FLEITH (P.N.C., FLORAC), les Fédérations de pêche des Pyrénées-Orientales et des Alpes-Maritimes, et les gardes chefs et fédéraux du C.S.P. Enfin, F. Bonhomme et F. Catzeflis ont fait une lecture critique du manuscrit.

\section{BIBLIOGRAPHIE}

ALMAÇA C., 1968. Révision critique de quelques types de cyprinidés d'Europe et d'Afrique du Nord des collections du Muséum National d'Histoire Naturelle. Bull. Mus. Nat. Hist. Nat. $2^{\mathrm{e}}$ sèrie, T 40, n०6, 1968 (1969): 1116-1144. 
ALMAÇA C., 1971. Sur la collection de Barbeaux (genre et sous-genre Barbus) iberiques et Nordafricains du Forschungsinstitut Senckenberg (Frankfurt A.M.). Arq. Mus. Boc ( ${ }^{\circ}$ série) vol. III, $21: 1-5$

ALMAÇA C., 1976. La spéciation chez les Cyprinidés de la Péninsule Ibérique. Rev. Trav. Inst. Pêches marit., $40: 399-411$.

ALMAÇA C., 1981. La collection de Barbus d'Europe du Muséum National d'Histoire Naturelle (Cyprinidae, Pisces). Bull. Mus. Nat. Hist. Nat., Paris, $4^{\text {e }}$ sér., 3, Section A, 1 : 277-307.

ALMAÇA C., 1984. Form relationships among Western Paleartic species of Barbus (Cyprinidae, Pisces). Arq. Mus. Boc (Série A), Vol. II, 12 : 207-248.

BANARESCU P., 1964. Fauna Republicii Populare Romine. Pisces. Osteichthyes. Ed. Acad. Repub. Pop. Romine, Bucuresti, $13: 959$ pp.

BANARESCU P. 1973. Origin and affinities of the freswater fish fauna of Europe. Ichthyologia, 5:1-8.

BERG L.S., 1932. Ubersicht der Verbretung der Susswasserfische Europas. Zoogeographica, $1: 107-208$.

BERREBI P., 1981. Contribution à l'étude du sous-genre labeobarbus (genre Barbus, Poissons, Cyprinidés) au Maroc. Bull. Inst. Sc. Rabat., 5 : 59-72.

DARLINGTON P.J., 1948. The geographical distribution of cold-blooded vertebrates. Quart. Rev. Bio., 23 : 105-123.

DARLINGTON P. J., 1957. Zoogeography: The geographical distribution of animals. Wiley ed., London.

DOTTRENS G., 1952. Poissons d'eau douce (2 volumes). DELACHAUX et NIESTLE Eds., Neuchâtel.

HAFEZ R., 1981. Études sur la cytogénétique et l'évolution génétique chez quelques Cyprinidés de la région Midi-Pyrénées. Thèse Inst. Nat. Polytechnique Toulouse : $362 p$

KARAMAN M.S., 1971. Süsswasserfische der Türkei. 8. Teil. Revision der Barben Europas, Vorderasiens und Nodarfrikas. Mitt. Hamburg Zool. Mus Inst., 67 : 175-254.

KOLLER O., 1926. Drei Fischarten aus Albanien, darunter eine bischer unbeschriebene Varietät von Barbus meridionalis Risso. Zool. Anzeiger., 65 : 315-320.

LADIGES W. \& VOGT D., 1965. Die Süsswasserfische Europas bis zum Ural un Karpischen Meer. Paul PAREY, Hamburg-Berlin.

MORETTI J., BROUSSIER G. \& JAYLE M.F., 1957. Réalisation technique et premières applications de l'électrophorèse sur gel d'amidon. Bull. Soc. Chim. Biol., 39 : 593-605.

PASTEUR N., AUTEM M., PICHOT P. \& GOUCHA M., 1985. Structure génétique de la Sole (Solea vulgaris, Quensel, 1806 ; Téléostéens, Soléidés). Premier catalogue de polymorphismes biochimiques accessibles par l'électrophorèse en gel d'amidon. Rev. Trav. Inst. Pêches marit., $47: 37-54$ (1985).

SHAW C.R. \& PRASAD R., 1970. Starch gel electrophoresis of enzymes. A compilation of recipes. Biochem. Genet., $4: 297-320$.

SOFRADZIJA A. \& BERBEROVIC L., 1973. The chromosome number of Barbus meridionalis petenyi Heckel (Cyprinidae, Pisces). Bull Sci. Acad. RSF Yougol., 18 : 77-88.

SPILLMAN J., 1961. Poisson d'eau douce. Faune de france $n^{\circ} 65$. LECHEVALIER, Paris.

STEPHANIDIS A., 1971. Poissons d'eau douce du Péloponnèse. Biol. Gallo-Hellenica, 3 : $163-212$.

TRIANTAPHYLLIDIS C.D., DAMIANAKIS H., ECONOMIDIS P.S. \& KARAKOUSIS J., 1981. Genetic variation in greek barbel population. I. Esterases, $\mathrm{LDH}, \mathrm{MDH}, \mathrm{ME}$ and PGM in Barbus meridionalis (Pisces, Cyprinidae). Comp. Biochem. Physiol., 70 B : 289-293.

VUKOVIC T. \& IVANOVIC B., 1971. Slatkovodne ribe Jogoslavije. Zemalski Muzej Bih, Sarjevo.

WOLF U., RITTER H., ATKIN N. and OHNO S., 1969. Polyploidization in the fish family Cyprinidae, order Cypriniforms. I : DNA contenand chromosome sets in various species of Cyprinidae. Humangenetik, $7: 240-244$. 